\title{
Joint replenishment and delivery problem with fuzzy chance constraint resouce restriction
}

\author{
Chenyan Li, Tianwei Zhang*and Shujie Yin \\ School of Computer Science and Technology, Harbin University of Science and Technology, \\ Harbin 150080, China
} Keywords: Supply chain management, Joint replenishment and delivery problem, Fuzzy chance
constraint, Triangle fuzzy number, Optimization algorithm.

\begin{abstract}
The joint replenishment and delivery problem with fuzzy chance constraint resource restriction is considered. In the proposed approach, the objective function is to minimize the ordering cost, the inventory holding cost, the transportation cost and the customer waiting cost. Mathematical model is developed, and differential evolution (DE) algorithm is proposed to solve the problem. The performance of the algorithm is then verified and test under the indirect group strategy for 1600 randomly generated problems. The results show the robust performance of the proposed algorithms.
\end{abstract}

\section{Introduction}

In recent years, many companies have realized that significant cost saving can be achieved by integrating inventory control and delivery policies throughout their supply chains, that is, the joint replenishment and delivery scheduling (JRD) policy. It is assumed in the JRD that for each item $i$, its demand rate $D_{i}$ is fixed, and each unit incurs a holding cost $h_{i}$ per unit time. A replenishment is made at regular time intervals (every $T$ units of time) and each item has a replenishment quantity sufficient to last for exactly an integer multiple $k_{i}, i=1,2, \ldots, n$ of $T$. The decision variables are $T$ and $k_{i}$ and the objective is to find a proper combination $\left(T, k_{i}\right)$ so that total cost is as low as possible.

Moon et al. [1] developed joint replenishment and consolidated freight delivery policies for a third party warehouse with deterministic demand rates in a supply chain. Cha et al. [2] dealt with the JRD model of the one-warehouse $n$-retailer system and suggested a flexible policy for a warehouse. Wang et al. [3] studied a joint replenishment and delivery scheduling in which a central warehouse serves n-retailers in the presence of vague operational conditions such as ordering cost and inventory holding cost, and the fuzzy membership function is approximated using piecewise linear functions based on alpha level sets.

The limitation common in all JRD models mentioned above is that capacities are assumed to be unlimited. In fact, there are many resource restrictions in real supply chain systems. Van Eijs [4] has derived a heuristic algorithm for the inventory control policy with one resource restriction. Uncertainties in JRD modeling have been studied by stochastic approach and fuzzy methods. As a kind of stochastic programming, chance constrained programming provides a means of allowing the decision maker to consider objectives in terms of the probability of their attainment. The term chance is represented by the probability that the constraints are satisfied $[5,6]$.

Jana and Sharma present a procedure for solving chance constrained programming problem, and a stochastic simulation-based genetic algorithm is developed to handle the randomness [7]. The differential evolution (DE) algorithm is one of the latest evolutionary optimization methods proposed by Storn and Kenneth [8] for complex continuous non-linear functions. DE has been successfully applied to resolve optimization problems, which exhibits remarkable performance in optimizing [9].

The rest of this paper is organized as follows. Section 2 introduces the mathematical model of fuzzy chance constraint JRD. Section 3 develops the DE algorithm and presents the procedure to solve the problem. Section 4 is an example for the DE algorithm. Section 5 summarizes the conclusions of the present work. 


\section{Mathematical model of the problem}

The joint replenishment and delivery problem with fuzzy chance constraint resource restriction (which is abbreviated as FCR-JRD for the rest of the paper) is the multi-item inventory problem of coordinating the replenishment and delivery of a group of items that may be jointly ordered from a single supplier under resource restrictions.

The total relevant cost per unit time is given by:

(FCR-JRD)

$\min T C(T, K, F)$.

subject to

$$
\begin{aligned}
& \operatorname{TC}(T, K, F)=\frac{S^{W}+\sum_{i=1}^{n} \frac{s_{i}^{W}}{k_{i}}}{T}+\sum_{i=1}^{n} \frac{\left(f_{i}-1\right) k_{i} T D_{i} h_{i}^{W}}{2 f_{i}}+\sum_{i=1}^{n} \frac{f_{i} s_{i}^{C}}{k_{i} T}+\sum_{i=1}^{n} \frac{k_{i} T D_{i} w_{i}^{C}}{2 f_{i}} . \\
& \operatorname{Pos}\left\{\xi \mid \sum_{i=1}^{n} D_{i} k_{i} T b_{i}-\xi \leq 0\right\} \geq \alpha . \\
& k_{i} \in Z^{+}, f_{i} \in Z^{+}, T \in R^{+}, i=1,2, \ldots n .
\end{aligned}
$$

The following notations are defined:

$i \quad$ index of item, $i=1,2, \ldots, \mathrm{n}$

$D_{i} \quad$ demand rate of item $i$

$S^{w} \quad$ warehouse's major ordering cost

$S_{i}^{w} \quad$ warehouse's minor ordering cost of item $i$

$h_{i}^{w} \quad$ warehouse's inventory holding cost of item $i$ per unit per time

$s_{i}^{c} \quad$ warehouse's outbound transportation cost of item $i$

$w_{i}^{c} \quad$ retailer's inventory holding cost of item $i$ per unit per unit time

$T$ warehouse's basic cycle time (decision variable)

$k_{i} \quad$ integer number that decides the replenishment schedule of item $i$ (decision variable)

$f_{i}$ integer number that decides the outbound schedule of item $i$ (decision variable)

Eq. 1 is the total cost to be minimized, that is the sum of the ordering cost (major and minor), the inventory holding cost, the outbound transportation cost, and the customer waiting cost. The resource constraint is the limit on capital that can be invested, as given by Eq. 3, is a fuzzy parameter with confidence level $\alpha$. Eq. 4 indicate that $k_{i}^{\prime}$ s and $f_{i}^{\prime}$ s are positive integer number and $T$ is positive real number.

The resource restriction $\xi$ is a triangle fuzzy number, whose membership function is Eq. 5 .

$$
\mu(x)=\left\{\begin{array}{l}
\frac{x-r_{1}}{r_{2}-r_{1}}, r_{1} \leq x \leq r_{2} \\
\frac{x-r_{3}}{r_{2}-r_{3}}, r_{2} \leq x \leq r_{3} . \\
0, \quad \text { otherwise }
\end{array}\right.
$$

To solve the unconstrained JRD, Moon et al. [11] used Eq. 6 as the optimal $T$ in their algorithm. 


$$
T^{*}=\left[2\left(S^{W}+\sum_{i=1}^{n} \frac{s_{i}^{W}+f_{i} s_{i}^{C}}{k_{i}}\right) / \sum_{i=1}^{n} k_{i} D_{i}\left(h_{i}^{W}+\frac{w_{i}^{C}-h_{i}^{W}}{f_{i}}\right)\right]^{1 / 2} .
$$

The optimality condition of $k_{i}$ is:

$$
k_{i}\left(k_{i}-1\right) \leq \frac{2\left(s_{i}^{W}+f_{i} s_{i}^{C}\right)}{T^{2} D_{i}\left(h_{i}^{W}+\frac{w_{i}^{C}-h_{i}^{W}}{f_{i}}\right)} \leq k_{i}\left(k_{i}+1\right) .
$$

Similarly, the optimality condition of $f_{i}$ is :

$$
f_{i}\left(f_{i}-1\right) \leq \frac{k_{i}^{2} T^{2} D_{i}\left(w_{i}^{C}-h_{i}^{W}\right)}{2 s_{i}^{C}} \leq f_{i}\left(f_{i}+1\right) \text {. }
$$

\section{Differential evolution algorithm}

In order to solve FCR-JRD, in this section, we present a differential evolution (DE) algorithm approach.

3.1 Representation and initialization. In the FCR-JRD model, the basic cycle $T$ and $n$ integer $k_{i}$ 's and $f_{i}$ 's have to be decided for solving the problem. In the DE, $k_{i}$ 's and $f_{i}$ 's are searched through the operations of DE and the basic cycle $T$ is determined through the optimality condition of $T$. For the given $k_{i}$ 's and $f_{i}$ 's , the optimal $T^{*}$ can be easily obtained from the Eq.6. So, in our study, we use $2 n$ random number representation for $n k_{i}$ 's and $n f_{i}$, s, because it is very easy to decode our chromosome to a feasible solution.

The initial population is created by assigning random integer values of the decision variable. Each individual is generated by Eq. 9 .

$$
x_{i j}=\operatorname{rand}(), i=1,2, \ldots, P O P S I Z E, j=1,2, \ldots, D \text {. }
$$

Where POPSIZE is the number of individuals; $D$ is the dimension of each individuals; rand () is a function which generates a uniform distribution rand number in range $\left[k_{i}^{L B}, k_{i}^{U B}\right]$ or $\left[f_{i}^{L B} f_{i}^{U B}\right] . k_{i}^{L B}$ and $k_{i}^{U B}$ are the lower and upper bound of $k_{i} . f_{i}^{L B}$ and $f_{i}^{U B}$ is the lower and upper bound of $f_{i}$, respectively.

$T_{\max }$ and $T_{\min }$ were defined in Eq. 10 and Eq. 11.

$$
\begin{aligned}
& T_{\max }=\left[2\left(S^{W}+\sum_{i=1}^{n} s_{i}^{W}\right) / \sum_{i=1}^{n} D_{i} h_{i}^{W}\right]^{1 / 2} . \\
& T_{\min }=\min \left(2 s_{i} / D_{i} h_{i}^{W}\right) .
\end{aligned}
$$

3.2 Mutation. For each target individual $X_{i}=\left\{x_{i 1}, x_{i 2}, \ldots, x_{i D}\right\}, i=1,2, \ldots, P O P S I Z E$, a mutant new individual $V_{i}$ is generated.

With randomly chosen integer indexes; $F \in[0,2]$ is a real number called mutation factor used to control the amplification of the differential variation.

3.3 Crossover. To complement the differential mutation search strategy, DE employs uniform crossover, which also known as binomial method, to enhance the potential diversity of the population. The crossover operator implements a discrete recombination of the trial individual $V_{i}$ and the parent individual $X_{i}$ to produce the offspring $X_{i}^{\text {new }}$.

3.4 Selection. The selection operator is to determine whether the target (parent) or the new vector (offspring) survives to the next generation. If a new vector, $X_{i}^{\text {new }}, i=1,2, \ldots, P O P S I Z E$, has a 
smaller evaluation function value (total cost) than its garget vector, $X_{i}$, it is copied to the next generation; otherwise, it is the target vector that passes to the next generation.

3.5 Constraint check. The main idea of constraint check is to keep all individuals within the feasible solution set. All the new individuals generated by initialization, mutation and crossover processes must be checked. A function constraint_check() is designed for the algorithm, the output value 1 means that the individual is feasible, 0 infeasible.

The pseudo code of constraint check is as follows:

constraint_check ()

Step 1 Generate $\xi^{0}$ uniformly from the $\alpha$-cut set of fuzzy vector $\xi$.

Step 2 If $\sum_{i=1}^{n} D_{i} k_{i} T b_{i}-\xi^{0} \leq 0$, return 0 .

Step 3 Return 1.

3.6 Stop criterion. The termination condition is to stop if no improvement of fitness function is made in 50 generations.

\section{Computation experiments}

A numerical example will be employed to illustrate the proposed DE algorithm. We use the numerical example of Moon et al. [6] and Wang et al. [8]. The data for this example are given in Table 1. We also assume $S^{W}=200$. Each replenishment cycle available funds limit is $B$. Fuzzy resource restriction $\xi$ is triangle fuzzy number with membership function $\mu(x), r_{1}=0.9 B, r_{2}=B$, and $r_{3}=1.1 B$, respectively. $\alpha=0.9$ is a predetermined confidence level.

Table 1. Parameter values for the example

\begin{tabular}{lllllll}
\hline Item $i$ & 1 & 2 & 3 & 4 & 5 & 6 \\
\hline$D_{i}$ & 10000 & 5000 & 3000 & 1000 & 600 & 200 \\
$s_{i}{ }^{W}$ & 45 & 46 & 47 & 44 & 45 & 47 \\
$h_{i}{ }^{W}$ & 1 & 1 & 1 & 1 & 1 & 1 \\
$s_{i}{ }^{C}$ & 5 & 5 & 5 & 5 & 5 & 5 \\
$w_{i}{ }^{C}$ & 1.5 & 1.5 & 1.5 & 1.5 & 1.5 & 1.5 \\
$b_{i}$ & 6.25 & 6.25 & 6.25 & 6.25 & 6.25 & 6.25 \\
\hline
\end{tabular}

The DE algorithm for FCR-JRD was coded in C++ language and running in a PC with Intel i5 CPU and 4G RAM. The computational result was shown in Table 2.

Table 2. Solutions and total costs for numerical example

\begin{tabular}{cllllc}
\hline$B$ & $T$ & $k_{i}$ & $f_{i}$ & $T C$ & $\begin{array}{c}\text { Running } \\
\text { time[s] }\end{array}$ \\
\hline 25000 & 0.1802 & $1,1,1,2,2,4$ & $2,3,2,3,1,2$ & 4903.66 & 545 \\
26000 & 0.1845 & $1,1,1,2,2,4$ & $4,2,2,2,2,2$ & 4842.97 & 301 \\
27000 & 0.1881 & $1,1,1,2,2,4$ & $4,3,2,3,2,2$ & 4828.89 & 73 \\
28000 & 0.1881 & $1,1,1,2,2,4$ & $4,3,2,3,2,2$ & 4828.89 & 21 \\
29000 & 0.1881 & $1,1,1,2,2,4$ & $4,3,2,3,2,2$ & 4828.89 & 6 \\
\hline
\end{tabular}

The result shows that with tighter resource restriction, the total cost was higher, for $B=25000$ and 26000 , respectively. The DE algorithm spends significantly more CPU time to obtain feasible solution with smaller $B$.

\section{Conclusion}

This paper focuses on the constrained joint replenishment and delivery problem with fuzzy chance constraint resource restriction, and developing efficient algorithms to solving it. We introduced the DE algorithm to handle the constraints in JRD. Using comprehensive computational 
experiments, we have shown the performance of the proposed algorithm. Furthermore, DE is more suitable for solving constrained JRD. Future research could be the incorporation of uncertainty issue with more constrained, for example uncertain demand.

\section{Acknowledgements}

This work was supported by the Education Department of Heilongjiang Province, China, under Grant No.12541142.

\section{References}

[1] I. K. Moon, B. C. Cha and C. U. Lee, The joint replenishment and freight consolidation of a warehouse in a supply chain, International Journal of Production Economics. 133 (2011) 344-350.

[2] B. C. Cha, I. K. Moon and J. H. Park, The joint replenishment and delivery scheduling of the one-warehouse, n-retailer system, Transportation Research and E-Logistics. 44 (2008) 720-730.

[3] L. Wang, C. X. Dun, C. G. Lee, Q. L. Fu and Y. R. Zeng, Model and algorithm for fuzzy joint replenishment and delivery scheduling without explicit membership function, International Journal of Advanced Manufacturing Technology. 66 (2013) 1907-1920.

[4] M. J. G. Van Eijs, A note on the joint replenishment problem under constant demand, Journal of the Operational Research Society. 44 (1993) 185-191.

[5] B. Liu and K. Iwamura, Chance constrained programming with fuzzy parameters, Fuzzy Sets and Systems. 94 (1998) 227-237.

[6] B. Liu and K. Iwamura, A note on chance constrained programming with fuzzy coefficients, Fuzzy Sets and Systems. 100 (1998) 229-233.

[7] R.K. Jana and D. K. Sharma, Genetic algorithm-based fuzzy goal programming for class of chance-constrained programming problems, International Journal of Computer Mathematics. 87 (2010) 733-742.

[8] R. Storn and P. Kenneth, Differential Evolution - A simple and efficient heuristic for global optimization over continuous spaces, Journal of Global Optimization. 11 (1997) 341-359.

[9] A. Musrrat, P. Millie and A. Ajith, A simplex differential evolution algorithm: Development and applications, Transactions of the Institute of Measurement and Control. 34 (2012) 691-704. 